\title{
An Intelligent Video System for Vehicle Localization and Tracking in Police Cars Amirali Jazayeri ${ }^{\dagger}$, Hongyuan $\mathrm{Cai}^{\dagger}$, Jiang Yu Zheng ${ }^{\dagger}$, Mihran Tuceryan $^{\dagger}$, Herbert Blitzer* ${ }^{\dagger}$ Department of Computer Science, Indiana University Purdue University Indianapolis, 723 W. Michigan St, Indianapolis, IN 46202 \\ * Indiana Forensic Institute, 338 S. Arlington Ave., Indianapolis, IN 46219 \\ jazayera@iupui.edu, hocai@iupui.edu, jzheng@cs.iupui.edu, tuceryan@iupui.edu, hblitzer@ifi-indy.org
}

\begin{abstract}
This work aims at real-time in-car video analysis to detect several critical events in order to alarm and assist police action. Particularly, detecting a tracked or stopped vehicle is a crucial task for further examination of suspects, protecting police safety, and remote monitoring from police station. This paper describes a comprehensive approach to localize targeted vehicles in the video under various environments and illumination conditions. The extracted geometry features on the moving objects and background are dynamically projected onto a 1D profile and are constantly tracked. We rely on temporal information of features for vehicle identification, which compensates for the complexity of vehicle shapes, colors and types. We investigated videos of day and night, and different types of roads, proving that our employed approach is robust and effective.
\end{abstract}

\section{Categories and Subject Descriptors}

I.4.8 [Scene Analysis]: Object recognition, Motion, Tracking, Time-varying imagery; I.4.9 [Applications]; I.2.10 [Vision and Scene Understanding]: Video analysis

\section{General Terms}

Algorithms, Performance, Experimentation

\section{Keywords}

In-Car Video, Tracking, Vehicle Detection, Spatial-Temporal Image, 1D projection, Intelligent Video System

\section{INTRODUCTION}

The use of video in police cars was found to have significant value and the number of such installed systems is increasing [1] The study points out, besides other things, that in-car video can enhance officer safety; improve agency accountability, and advance prosecutions and case resolution.

The prerequisites for an in-car monitoring application are the detection, localization, and tracking of vehicles. In this paper, we

Permission to make digital or hard copies of all or part of this work for personal or classroom use is granted without fee provided that copies are not made or distributed for profit or commercial advantage and that copies bear this notice and the full citation on the first page. To copy otherwise, or republish, to post on servers or to redistribute to lists, requires prior specific permission and/or a fee.

SAC'09, March 8-12, 2009, Honolulu, Hawaii, U.S.A.

Copyright 2009 ACM 978-1-60558-166-8/09/03 ...\$5.00. propose a novel approach for detecting and tracking vehicles captured by a vehicle-borne camera. This system could potentially be used as the backbone of the safety application that is connected to officer's microphone, GPS system, and police car's radio.

There have been a large number of papers describing vision system for car detection $[2,3,4]$. In these cases car detection is possible through image segmentation based on movement.

In this paper we propose a car detection method by a vehicleborne camera intended for situations where no prior knowledge is provided for targeting vehicles in the front. We track and identify dynamic vehicles ahead of the police car under a changing environment and illumination.

\section{FEATURE DETECTION}

Our goal is segmenting the vehicles close to the camera from the background. This is difficult due to the complex nature of scenes; it contains other moving vehicles, complicated shape and texture of ever changing background. The presence of specular reflection and shadows on the metallic surfaces of cars make the detection unreliable. Cars with colors similar to the background meld into their surroundings and become indistinguishable. Figure 1 shows several examples of vehicles on roads.

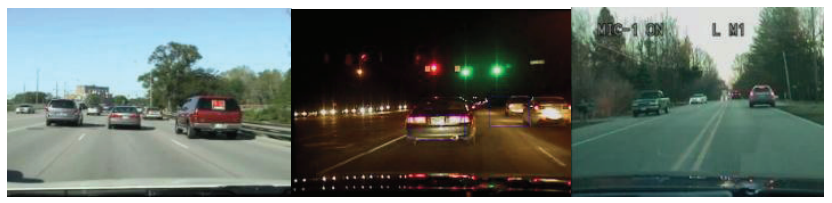

Figure 1 Vehicles in front of the police car.

In order to avoid including the sky, high buildings, and the police car dashboard in the vehicle detection, we design a mask to filter out the low probability regions of vehicle presence. Vehicles appear most frequently in the image across the projected horizon, meaning that the horizon line passes through the vehicle. The mask is shown as in Figure 2.
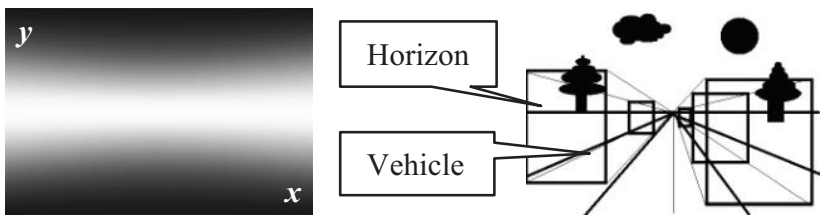

Figure 2 Mask the image according to the appearing locations of vehicles. The intensity corresponds to weight of multiplication. 
Depending on the lighting conditions, we extract horizontal line segments, intensity peaks, and corner points [5] in each frame.

\section{TEMPORAL TRACKING OF VEHICLES}

We project features extracted in the video frames vertically onto the $\mathrm{x}$-axis to generate a $1 \mathrm{D}$ representation of the frame content. The 1D projections stacked in time result in a spatio-temporal feature traces. This representation is computed by:

$$
T_{l}(x, t)=\sum_{y=-h / 2}^{h / 2} w(x, y) C(x, y, t)
$$

where $C(x, y, t)$ is 1 if $(x, y)$ is on a feature in frame $t$, and 0 otherwise. An example is shown in Figure 3, in which the bright stripes show the motion of vehicle.
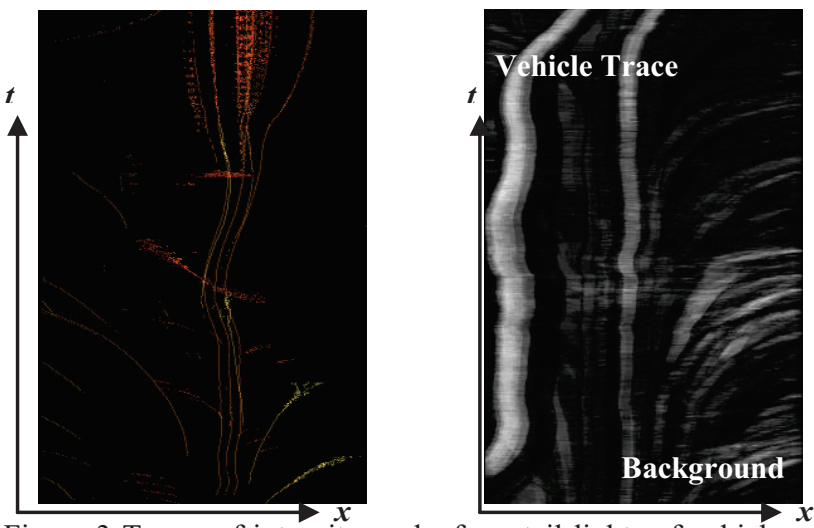

Figure 3 Traces of intensity peaks from tail lights of vehicle and traces of horizontal line segments on vehicles.

By using the properties of motion, we can distinguish different traces in the spatio-temporal representation to help our vehicle identification task. According to the motion properties, the background scenes captured from a forward-looking vehicleborne camera result in a translation relative to the camera. Their image velocity is high at nearby points on the ground and buildings passing by, and is low at the distant scenes down further ahead. The feature points follow motion traces of hyperbolas expanding from the vanishing point on the road (Focus of Expansion), if the camera motion is a pure translation.

This can be observed in the spatio-temporal image where the image velocity of scenes corresponds to the orientation of the traces; the higher the speed, the more the trace is slanted and eventually exits the image from the left or right side. Therefore, background scenes move sideways with an increasing speed. Using this property of background motion, we can detect the cars which leave consistent traces in the spatio-temporal image.

A tracking history is created for each trace. By defining limits for the last update time and tracking duration we are able to separate the background from the vehicle traces successfully. We have assumed that a target vehicle will not vanish from the field of view rapidly.

\section{EXPERIMENTAL RESULTS}

We have tested our method on videos collected from cameras onboard police vehicles, and videos recorded using a commercial camcorder. These videos contain scenes shot during dusk, dawn, day, and night. In our tests, we found that our method has $89.7 \%$ success rate in detecting vehicles. In most cases of correct detection, the duration of the tracking lasts as long as the vehicle remains in the scene. If a vehicle moves too far from the police car, the tracking stops. Example results of detected cars are shown in Figure 4.

\section{CONCLUSION}

In this paper, we presented one component of an intelligent video system to be deployed in police cars. The detection and tracking of the target vehicles is done based on properties in a reduced spatio-temporal representation. This method has successfully detected and localized cars in a substantial percentage of our test videos shot in real situations. This system will eventually be utilized in the detection of critical events during a police stop of a targeted car.

\section{ACKNOWLEDGMENTS}

This work was supported by the US National Institute of Justice grant \#2007-DE-BX-K007.

\section{REFERENCES}

[1] Baker, W.G. et al. IACP report to National Institute of Justice entitled, The Impact of Video Evidence on Modern Policing (NIJ grant \#2001-CK-WX-0157), 2004.

[2] Alonso, D.; Salgado, L.; Nieto, M.; Robust Vehicle Detection through Multidimensional Classification for on Board Video Based Systems, In IEEE International Conference on Image Processing (ICIP). Volume 4, 321 324, Sept. 16-19 2007.

[3] Demonceaus C., Potelle A., and Kachi-Akkouche D., Obstacle detection in a road scene based on motion analysis, IEEE Trans. On Vehicular Technology, 53(6), 72-77, 2004.

[4] Kate, T.K. ten; Leewen, M.B. van; Moro-Ellenberger, S.E.; Driessen, B.J.F.; Versluis, A.H.G.; Groen, F.C.A.; Mid-range and distant vehicle detection with a mobile camera, In IEEE Intelligent Vehicles Symposium, 72-77, June 2004.

[5] C. Harris and M. Stephens, A combined corner and edge detector. In Proceedings of the 4th Alvey Vision Conference, 147-151, 1988.

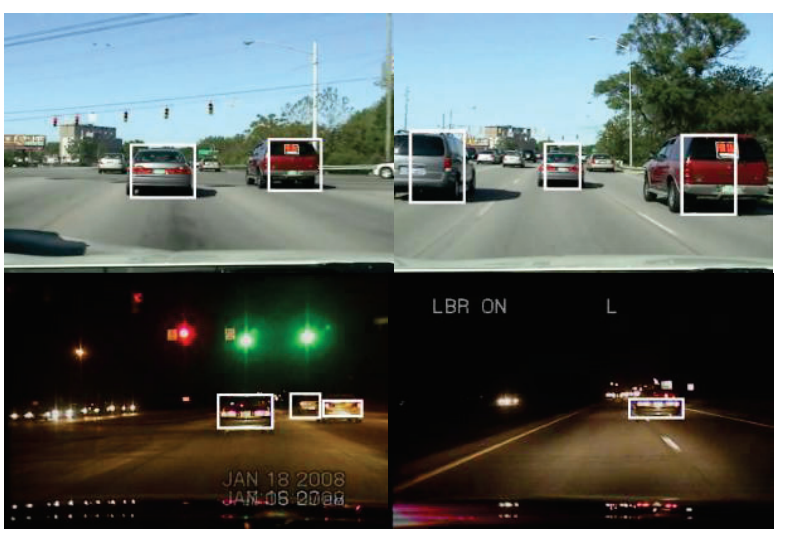

Figure 4 The result of detected and localized vehicles in daytime and nighttime sequences. 